\title{
High-efficiency Rectifier for Passive RF Energy Harvesting Devices
}

\author{
Yuchen Wang, Xiaohong Peng, Ligang Hou, Shuqin Geng \\ VLSI and System Lab, Beijing University of Technology, Beijing 100124, China
}

Keywords: differential rectifier, RF-DC converter, wireless power transmission, CMOS integrated circuit.

ABSTRACT: In this paper, a highly efficient differential rectifier for passive radio frequency (RF) energy harvesting device is designed. The circuit uses the differential-drive CMOS to reduce the RF energy loss caused by the MOS threshold voltage during rectification and improve the rectification efficiency of the circuit. The circuit was fabricated with a $0.35 \mu \mathrm{m}$ CMOS process and the overall RF-DC power conversion efficiency for a DC output power of $160 \mu \mathrm{W}(2.5 \mathrm{~V}$ and $64 \mu \mathrm{A})$ is about $50 \%$. When high voltage is needed for stimulator output, the maximum efficiency for a $4 \mathrm{~V} \mathrm{DC}$ voltage reaches $66.5 \%$ at $3 \mathrm{dBm}$. The proposed rectifier allows the output voltage higher than $6 \mathrm{~V}$. It is suitable for such applications that require high RF input power such as implantable medical devices (IMDs) and passive industrial sensor.

\section{INTRODUCTION}

There is various RF power devices have been developed for different applications, such as implantable stimulators, industrial sensor, etc. For particular application which requires high level power consumption (higher than $1 \mathrm{~mW}$ ), the battery may lead many limitations. These problem can be overcome by using RF energy harvesting which is the process of receiving energy from the transmitter. Many researches transfer power by inductive coupling coils to achieve high power transmission efficiency [1]. It lead device's size considerably large. The UHF band $(860-960 \mathrm{MHz})$ is used to minimize device size and preform higher efficiency. The available power can be theoretically calculated by the Friis transmission equation

$$
P_{r}=G_{r} \times P_{E I R P} \times\left(\frac{\lambda}{4 \pi R}\right)^{2}
$$

Where $\lambda$ the wavelength of the EM wave, $R$ is the distance between transmitter and receiver, $P_{\text {EIRP }}$ is the effective isotropic radiation power of transmitter and $G_{r}$ is the receiver antenna gain. $P_{\text {EIRP }}$ is determined by regional regulations and $\mathrm{G}_{\mathrm{r}}$ is determined by the antenna design ( $2 \mathrm{dBi}$ for $\lambda / 2$ dipole antenna). For our cases, the available power received by antenna is assuming less than $5 \mathrm{dBm}$ and antenna gain is $1.64 \mathrm{dBi}$. The PCE is used to represent the rectifier's power efficiency and the detail will discuss in section III

In this work we design a high efficiency differential drive threshold voltage $\left(V_{t h}\right)$ cancellation rectifier circuit with regular voltage output and the circuit is compared with a conventional rectifier based on Dickson charge pump. Both of these circuits have been fabricated in $0.35 \mu \mathrm{m}$ CMOS process.

This paper is organized as follows. In Section II, we discuss the basic operation of conventional rectifier and how power loss in high voltage stimulator. The proposed rectifier circuit description is given in Section III. In Section IV we summarize our circuit performance and compare it to the control group result. Finally, Section V summarizes our overall conclusions. 


\section{CONVENTIONAL RECTIFIER CIRCUIT}

A conventional UHF rectifier is based on Dickson Charge Pump. In CMOS process use diodeconnected MOS take the place of diode, and two out-of-phase clocks is replaced by antenna amplitude. When GND is higher than $R F_{\text {in }}, \mathrm{M} 1$ is $\mathrm{ON}$ and $\mathrm{C} 1$ is charged. When $R F_{\text {in }}$ is high, M1 turns OFF while M2 turns $\mathrm{ON}$, the charge transfer from $\mathrm{C} 1$ to $\mathrm{C} 2$. To achieve high output voltage, multi-stage could be used. The output of an N stages Dickson's charge pump rectifier could be expressed as

$$
V_{D C}=2 \times N \times\left(V_{a m p}-V_{t h}\right)
$$

Where $\mathrm{N}$ is the number of stages, Vamp is the amplitude of RF input signal, and $V_{\text {th }}$ is the threshold of MOSFET. The diode-connect MOS can be represented as an equivalent resistance of resistor, and a single stage can be viewed as a RC network [3]. The delay of the stage could be expressed as

$$
\tau=k \times R_{e q} \times C_{c}
$$

Where $\mathrm{k}$ is the coefficient determined by the number of charge being transferred up to a proper percentage in a given period. With the frequency of clocks increased, the $R_{e q}$ shows a significant effect of the power convert efficiency of the rectifiers. To decrease the resistances effect, various rectifiers scheme has been proposed [4][5]. Those methods can improve efficiency by reduce the $R_{e q}$.

\section{PROPOSED DIFFERENTIAL-DRIVE RECTIFIER CIRCUIT}

To achieve low on-resistance and small leakage current, the differential-drive CMOS rectifier has been developed. The rectifier's unit stage is shown in Figure 1, the cross-coupled differential configuration with a bridge structure is driven by a differential signal and the DC output is the source of the PMOS.

The basic operation of those four transistors is described in following. $V_{X}$ and $V_{Y}$ are complementary signal. When $V_{X}$ is high and $V_{Y}$ is low, the MP1 and MN2 are on meanwhile MP2 and MN1 are off, the $V_{D C}$ equals $V_{X}$ and the chassis ground (GND) equals $V_{Y}$. Therefore the output voltage is $2\left(V_{a m p}-V_{t h}\right)$, where $V_{a m p}$ is the voltage amplitude of $V_{X}$ (or $V_{Y}$ ), $V_{\text {drop }}$ is the voltage drop cause by MOS resistance. For some applications, a high output voltage is needed. A multitude of stages could be series connected to get high voltage. The output voltage $V_{D C}$ is decided by forward current, reverse current and current flowing through the load. With the steady-state assumption, the current consumed on load $I_{L O A D}$ is equal to the charges transferred through the MP1 and MP2. The equation of $I_{L O A D}$ as follows:

$$
I_{L O A D}=\overline{I_{M P 1}+I_{M P 2}}=\frac{Q_{M P 1}+Q_{M P 2}}{T}
$$

Where $I_{M P 1}, I_{M P 2}$ is the current through pMOS, $Q_{M P 1}, Q_{M P 2}$ stand for the charges flowing through pMOS during the period $T$. The mainly analysis (take MP1 as an example) is shown as follows: follows:

When $V_{g s}$ of MP1 increases, the MP1 start to conduct and work in saturation region. The current is as

$$
I_{s a t}=K^{\prime} \frac{W}{2 L}\left(V_{g s}-V_{t h}\right)^{2}\left(1+\lambda V_{d s}\right)
$$

When the absolute value of $V_{g s}-V_{t h}$ decreases, MP1 work into linear region. The current is as follows: 


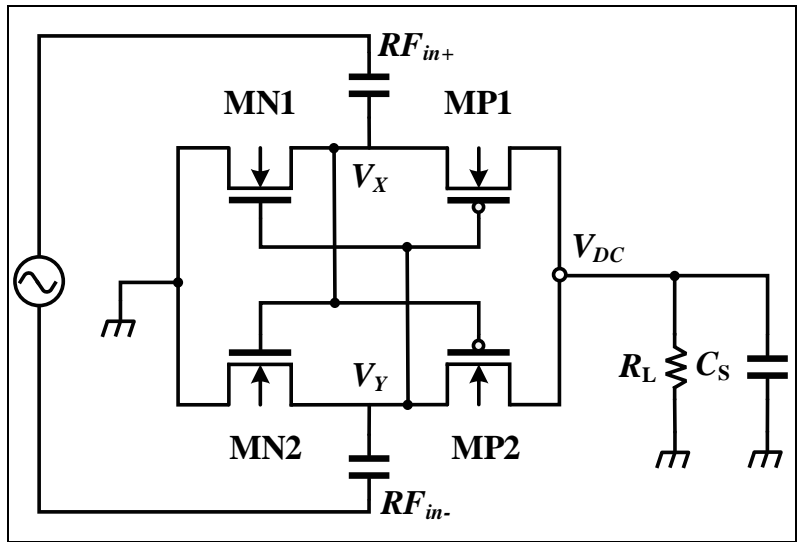

Figure 1. The proposed rectifier in this paper.

$$
I_{\text {linear }}=K^{\prime} \frac{W}{2 L}\left(V_{g s}-V_{t h}-\frac{1}{2} V_{d s}\right) V_{d s}
$$

When the transistors operate in weak-inversion region, lead a low PCE because of the higher channel resistance. The weak sub-threshold current is as follow:

$$
I_{\text {sub }}=I_{s 0}\left[1-\exp \left(-\frac{V_{d s}}{V_{t}}\right)\right] \exp \left(\frac{V_{g s}-V_{t h}-V_{o f f}}{n V_{t}}\right)
$$

Where $I_{S 0}$ is given by

$$
I_{s 0}=\mu_{0} \frac{W}{L} \sqrt{\frac{q \varepsilon_{s i} N_{c h}}{2 \phi_{s}}} V_{t}^{2}
$$

When the value of $V_{g s}$ and $V_{d s}$ is down to 0 , the transistor enters the cutoff region. Due to the leakage current existence, maximum PCE cannot be achieved. The leakage current can be defined by:

$$
I_{\text {leak }}=I_{s 0}\left[1-\exp \left(-\frac{V_{d s}}{V_{t}}\right)\right] \exp \left(\frac{-V_{\text {th }}-V_{\text {off }}}{n V_{t}}\right)
$$

By combining the above-described equations, the output voltage can be represent as a function three variables:

$$
V_{\text {out }}=f\left(\frac{W}{L}, R_{L}, V_{\text {th }}\right)
$$

In N-stage rectifier, if we define the output voltage the Nth stage by $V_{\text {out }}$, the output voltage can be solved by adding each stages

$$
V_{\text {out }}=V_{\text {out } 1}+V_{\text {out } 1}+\ldots+V_{\text {out } N}
$$

The N-stage rectifier's power convert efficiency is computed as

$$
\operatorname{PCE}(\%)=\frac{P_{D C}}{P_{\text {input }}} \times 100=\frac{P_{D C}}{P_{D C}+N \times P_{\text {LOSS }}} \times 100
$$

Where $P_{\text {input }}$ is the power received from antenna, $P_{D C}$ is the rectifier's output power, the $P_{\text {LOSS }}$ is given by

$$
P_{\text {LOss }}=P_{\text {sat }}+P_{\text {linear }}+P_{\text {sub }}+P_{\text {leak }}
$$

Where the $P_{\text {sat }}, P_{\text {linear }}, P_{\text {sub }}, P_{\text {leak }}$ is the power dissipation at separate region of transistors. 
The conversion efficiency does not take impedance mismatch into consideration, therefore it directly reflect the performance of the rectifier circuit rather than whole system. We use the circuit's efficiency as the main performance metric in this paper.

\section{DESIGN AND CIRCUIT PERFORMANCE}

A test chip was designed and fabricated with $0.35 \mu \mathrm{m}$ standard CMOS process. Figure 2 shows the micrograph of the fabricated test circuit, the active area is $140 \mu \mathrm{m} * 60 \mu \mathrm{m}$ not including the pad. The rectifier is compatible with standard CMOS processes. A conventional rectifier is fabricated as well. The off-chip series inductor is added to resonate out the input capacitance of the circuit. It provide $Q$ voltage boosting and it presents a higher voltage at the input port of the rectifier to achieve a better efficiency. The low-loss probing pads is essential to avoided substrate loss.

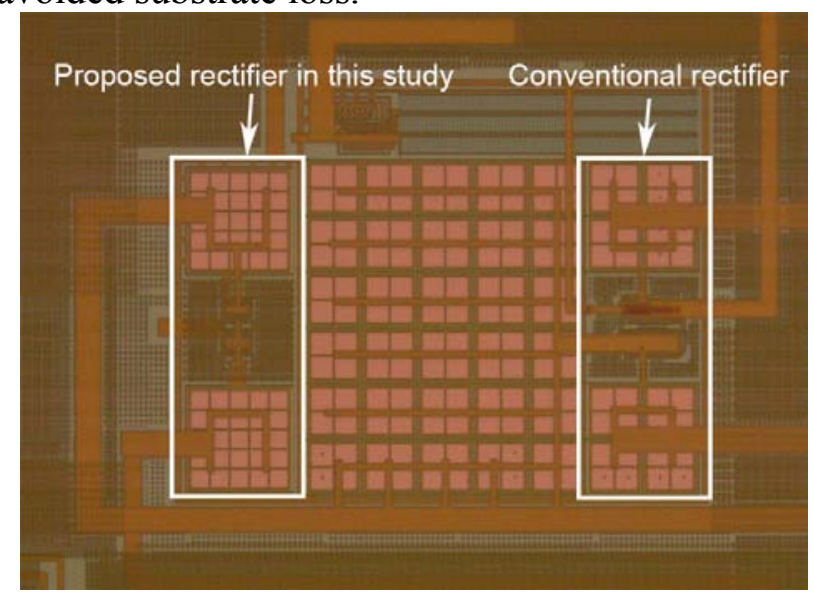

Figure 2. Micrograph of the fabricated circuits

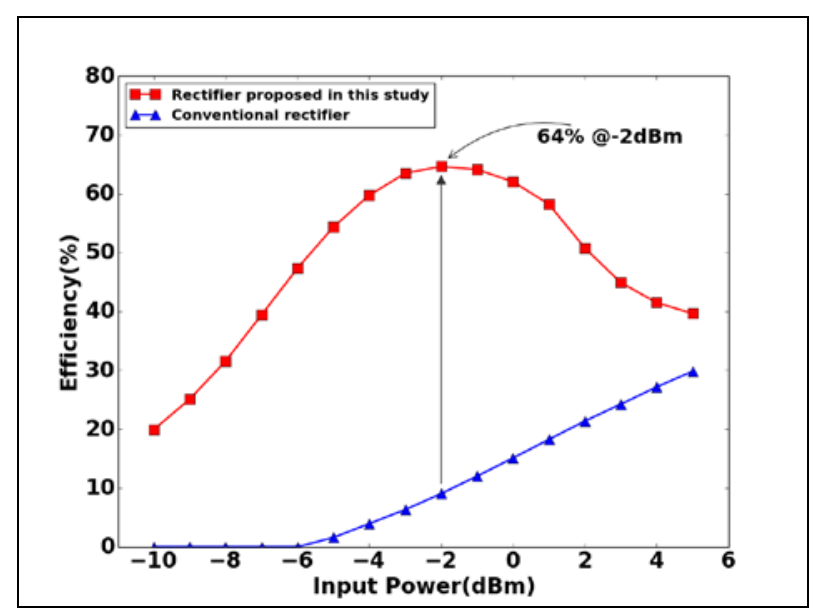

Figure 3. RF-DC converter efficiency versus the RF input power with different rectifier structure. 


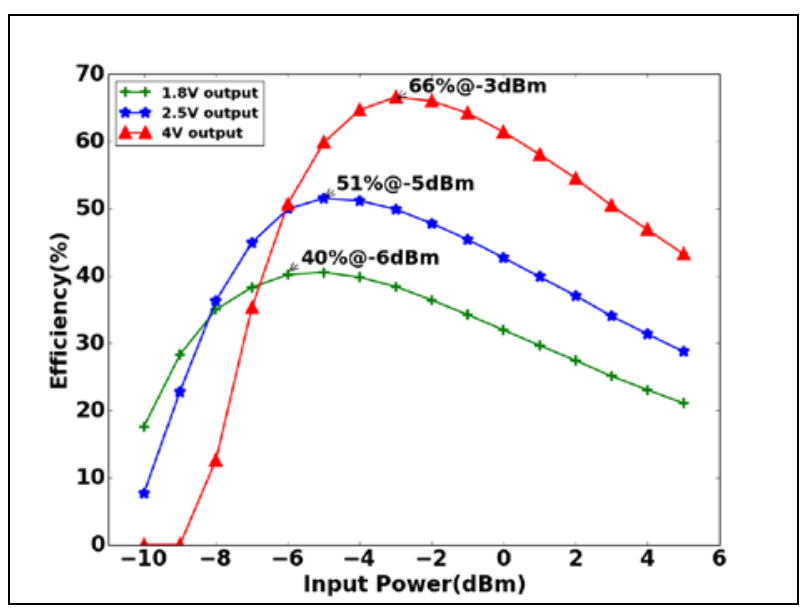

Figure 4. RF-DC converter efficiency versus the RF input power with different output DC voltage.

Table I. Performance Summary of CMOS UHF RF/DC Rectifiers

\begin{tabular}{|cccccc|}
\hline & This Work & {$[\mathbf{6}]$} & [7] & [8] & [9] \\
\hline Process & $0.35 \mu \mathrm{m}$ standard & $0.18 \mu \mathrm{m}$ & $65 \mathrm{~nm}$ RF & $0.35 \mu \mathrm{m}$ FeRAM & $0.25 \mu \mathrm{m}$ SOS \\
Stages & 5 & 24 & 5 & 1 & 1 \\
Frequency(MHz) & 915 & 900 & 900 & 953 & 915 \\
Pin(dBm) & -3 & -11 & +18 & -3.5 & -4 \\
PCE(\%) & 66 & 26.50 & 31.80 & 42 & 71.50 \\
\hline
\end{tabular}

The size was selected carefully for the balance between low equal on-resistance and low leakage current, therefore the width should be wide enough to achieve the proper $I_{d s}$. A smoothing polysiliconpolysilicon capacitor were placed at the output of the rectifier. The conventional CMOS rectifier were also fabricated with the same stages as the rectifier we proposed in this paper.

Figure 4 illustrate the PCE of both rectifier during sweep the input power from $-10 \mathrm{dBm}$ to $5 \mathrm{dBm}$ at $915 \mathrm{MHz}$ with different loads. As shown in this figure, the maximum RF-DC PCE of the rectifier for a DC output power of $162 \mu \mathrm{W}(2.5 \mathrm{~V}$ and $64 \mu \mathrm{A})$ is about $51.5 \%$ at $-5 \mathrm{dBm}$ input power. To provide high voltage for stimulator output, the maximum efficiency for a DC output voltage of $4 \mathrm{~V}$ is about $66 \%$ at $-3 \mathrm{dBm}$. For large input power, a large DC output voltage can be produced. It is noted that the output voltage exceeds breakdown voltage might lead a permanent damage to the devices unless high-voltage devices is used at the output. The performance of this chip is summarized in Table I [6]-[9] and the comparison between this work and some of the state of the art works is also presented.

\section{CONCLUDE}

We developed a high-efficiency CMOS rectifier circuits for wireless power neural tissue stimulator. The analytic expressions for the output voltage and power conversion efficiency were presented by reasonable approximations. The differential-drive architecture can reduce the effective threshold voltage of diode-connected MOS transistors and it is proved that the proposed circuit has a better performance of the PCE compare to the conventional rectifiers. An available DC power of at least $200 \mu \mathrm{W}$ is generated by the CMOS rectifier at a $\mathrm{RF}$ input power of $-4 \mathrm{dBm}$. In comparison with the conventional rectifier fabricated with same process the efficiency has been enhanced significantly. 


\section{ACKNOWLEDGMENTS}

This work is supported by the National Natural Science Foundation of China (No. 61204040, No.60976028), and Beijing Municipal Natural Science Foundation (No. 4152004).

\section{REFERENCES}

1. Young-Sik Seo, Minh Quoc Nguyen, Z. Hughes, S. Rao and J. C. Chiao, "Wireless power transfer by inductive coupling for implantable batteryless stimulators," Microwave Symposium Digest (MTT), 2012 IEEE MTT-S International, Montreal, QC, Canada, 2012, pp. 1-3.

2. R. E. Barnett, J. Liu and S. Lazar, "A RF to DC Voltage Conversion Model for Multi-Stage Rectifiers in UHF RFID Transponders," in IEEE Journal of Solid-State Circuits, vol. 44, no. 2, pp. 354-370, Feb. 2009.

3. F. Pan and T. Samaddar, Charge Pump Circuit Design. New York: McGraw-Hill, 2006, ISBN 0-08247045-X.

4. Koji Kotani and Takashi Ito, "High efficiency CMOS rectifier circuit with self-Vth-cancellation and power regulation functions for UHF RFIDs," Solid-State Circuits Conference, 2007. ASSCC '07. IEEE Asian, Jeju, 2007, pp. 119-122.

5. T. Umeda, H. Yoshida, S. Sekine, Y. Fujita, T. Suzuki and S. Otaka, "A 950-MHz rectifier circuit for sensor network tags with 10-m distance," in IEEE Journal of Solid-State Circuits, vol. 41, no. 1, pp. 35-41, Jan. 2006.

6. J. Yi, W. H. Ki and C. Y. Tsui, "Analysis and Design Strategy of UHF Micro-Power CMOS Rectifiers for Micro-Sensor and RFID Applications," in IEEE Transactions on Circuits and Systems I: Regular Papers, vol. 54, no. 1, pp. 153-166, Jan. 2007.

7. C. H. Tsai, I. N. Liao, C. Pakasiri, H. C. Pan and Y. J. Wang, "A Wideband $20 \mathrm{~mW}$ UHF Rectifier in CMOS," in IEEE Microwave and Wireless Components Letters, vol. 25, no. 6, pp. 388-390, June 2015.

8. H. Nakamoto et al., "A Passive UHF RFID Tag LSI with 36.6\% Efficiency CMOS-Only Rectifier and Current-Mode Demodulator in 0.35/spl mu/m FeRAM Technology," 2006 IEEE International Solid State Circuits Conference - Digest of Technical Papers, San Francisco, CA, 2006, pp. 1201-1210.

9. P. T. Theilmann, C. D. Presti, D. Kelly and P. M. Asbeck, "Near zero turn-on voltage high-efficiency UHF RFID rectifier in silicon-on-sapphire CMOS," 2010 IEEE Radio Frequency Integrated Circuits Symposium, Anaheim, CA, 2010, pp. 105-108. 